\title{
Scatter reduction for grid-less mammography using the convolution- based image post-processing technique
}

\author{
Elena Marimón ${ }^{* a, b}$, Hammadi Nait-Charif ${ }^{\mathrm{a}}$, Asmar Khan ${ }^{\mathrm{b}}$, Philip A. Marsden ${ }^{\mathrm{c}}$, Oliver Diaz ${ }^{\mathrm{d}}$ \\ ${ }^{\mathrm{a}} \mathrm{CDE}$ and Bournemouth University, Bournemouth Media School, BH12 5BB, Bournemouth, UK; \\ ${ }^{b}$ PerkinElmer Medical Imaging, 50-52 Wharf road, N1 7EU, Islington, London, UK; \\ 'UDA Ltd, 3 Skylines Village, Limeharbour, London, E14 9TS, UK; \\ ${ }^{\mathrm{d} U n i v e r s i t y ~ o f ~ G i r o n a, ~ C a m p u s ~ M o n t i l i v i, ~} 17071$ Girona, Spain.
}

\begin{abstract}
X-ray Mammography examinations are highly affected by scattered radiation, as it degrades the quality of the image and complicates the diagnosis process. Anti-scatter grids are currently used in planar mammography examinations as the standard physical scattering reduction technique. This method has been found to be inefficient, as it increases the dose delivered to the patient, does not remove all the scattered radiation and increases the price of the equipment. Alternative scattering reduction methods, based on post-processing algorithms, are being investigated to substitute anti-scatter grids. Methods such as the convolution-based scatter estimation have lately become attractive as they are quicker and more flexible than pure Monte Carlo (MC) simulations. In this study we make use of this specific method, which is based on the premise that the scatter in the system is spatially diffuse, thus it can be approximated by a two-dimensional low-pass convolution filter of the primary image. This algorithm uses the narrow pencil beam method to obtain the scatter kernel used to convolve an image, acquired without anti-scatter grid. The results obtained show an image quality comparable, in the worst case, to the grid image, in terms of uniformity and contrast to noise ratio. Further improvement is expected when using clinically-representative phantoms.
\end{abstract}

Keywords: X-ray, mammography, Monte-Carlo simulations, grid-less mammography, scatter radiation, convolution, pencil beam.

\section{INTRODUCTION}

One of the main challenges remaining in digital X-ray mammography is scattered radiation. Scattering reduces the quantitative usefulness of the mammogram, affecting the diagnosis of malignant lesions ${ }^{1,2,3}$.

Most of the planar mammography systems currently available in the market make use of anti-scatter grids as a technique to physically reduce the scattered radiation. Although this method helps to improve the image quality, it is not a complete solution. The grids do not block all the scattered radiation and attenuate a proportion of primary radiation, leading to an increase in the patient dose delivered, and add complexity and cost to the overall system ${ }^{1,4,5}$.

Image post-processing scatter reduction methods have emerged as a consequence of the limitations of the anti-scatter grids. Methods such as the convolution-based scatter estimation, have lately become attractive as they are faster and more flexible than direct Monte Carlo (MC) simulations ${ }^{4,6,7}$. This specific method is based on the idea that the scatter in the system is spatially diffuse, thus it can be approximated by a two-dimensional low-pass convolution filter of the primary image ${ }^{3}$.

In this paper, we compare the quality of clinical images acquired without anti-scatter grids that have been post-processed with the convolution-based scatter estimation method, and images acquired with anti-scatter grid.

\section{METHODOLOGY}

We assume that the image acquired from a digital mammography detector, $\mathrm{I}(\mathrm{x}, \mathrm{y})$, is the linear combination of the energy deposited by the primary and the scattered $\mathrm{X}$-ray photons, $\mathrm{P}(\mathrm{x}, \mathrm{y})$ and $\mathrm{S}(\mathrm{x}, \mathrm{y})$ respectively: 


$$
I(x, y)=P(x, y)+S(x, y) .
$$

The two dimensional (2D) description of the scatter can be seen as a low-pass filtered primary image ${ }^{8}$. However, the primary image is unknown and its determination is the principal objective of this study. To address this problem, we are following an approximation first introduced by Love and Kruger ${ }^{8}$ :

$$
S^{\prime}(x, y) \approx I(x, y) * h(x, y)
$$

where, $\mathrm{h}(\mathrm{x}, \mathrm{y})$ is a low-pass filter kernel that has to be determined.

With this approximation it is possible to calculate a new primary image, $\mathrm{P}^{\prime}(\mathrm{x}, \mathrm{y})$ :

$$
P^{\prime}(x, y)=I(x, y)-I(x, y) * h(x, y) .
$$

\subsection{Mammography system and software used}

Phantom images with and without anti-scatter grid were taken at Barts Health NHS Trust with a Hologic Lorad Selenia mammography system. The phantom used was a CIRS 010A test object. Both, grid and grid-less images, were obtained at constant exposure, $28 \mathrm{kV}$ and $100 \mathrm{mAs}$.

The GEANT4 toolkit (version 10.01.p02) was the software chosen to study the scattered radiation in the simulated mammography geometry ${ }^{9,10}$. The analysis was done with the aid of MATLAB, R2013b with Image Processing toolbox, and Image J, 1.47V.

\subsection{Definition of the Scatter Point Spread Function (SPSF)}

SPSF kernels were obtained from the MC simulation using a narrow X-ray beam which was represented by a normallyincident 2D spatial delta function, i.e. narrow pencil beam method ${ }^{7}$. Energy from scattered radiation (S) was binned into $35 \mu \mathrm{m}$ radius ( $\mathrm{r}$ ) concentric annuli (up to $175 \mathrm{~mm}$ ). These were then normalized to the sum of the primary image and the scatter radiation, $\mathrm{P}(0)+\mathrm{S}(\mathrm{r})$, and the area of the annuli, $\mathrm{A}(\mathrm{r})$, as described in ${ }^{11}$.

$$
\operatorname{SPSF}(r)=\frac{S(r)}{(P(0)+S(r)) A(r)}\left[\mathrm{mm}^{-2}\right]
$$

The final kernels were calculated from the scatter point spread function, see equation 4 . As the kernel was considered isotropic and the function was normalized by the area, the value of every pixel of the kernel could be defined by calculating its distance to the centre, interpolating the SPSF(r) function and multiplying by the area of the pixel, $70 x 70 \mu \mathrm{m}^{2}$ for the Hologic Lorad Selenia. The estimated primary image (P') was obtained following equations (2) and (3).

\subsection{Geometry used in this study}

The experimental geometry, i.e. Pencil Beam (PB) geometry, was defined to simulate the geometry of the Hologic Lorad Selenia mammography system, where the CIRS 010A phantom images were acquired. For the pencil beam methodology, the D-shaped CIRS phantom was changed to a cylindrical-shaped, simulating the scatter distribution at the centre of the phantom.

Table 1. Details of the materials and the thickness of the objects used in the validation and in the pencil beam geometry.

\begin{tabular}{|ccccc|}
\hline \multirow{2}{*}{ Object } & \multicolumn{2}{c|}{ Geometry Validation } & \multicolumn{2}{c|}{ Pencil Beam Geometry } \\
\cline { 2 - 5 } & Material & Thickness & Material & Thickness \\
\hline Breast compression paddle & PMMA & $2 \mathrm{~mm}$ & PMMA & $2.54 \mathrm{~mm}$ \\
Phantom tissue & 20\% glandular & $46 \mathrm{~mm}$ & $30 \%$ glandular & $40 \mathrm{~mm}$ \\
Phantom tissue & Skin layer & $2 \mathrm{~mm}$ & Adipose layer & $5 \mathrm{~mm}$ \\
Breast support paddle & PMMA & $2 \mathrm{~mm}$ & Carbon fibre & $2.54 \mathrm{~mm}$ \\
Air gap & Air & $13 \mathrm{~mm}$ & Air & $17.46 \mathrm{~mm}$ \\
Body & Water & $300 \mathrm{~mm}$ & No body & No body \\
\hline
\end{tabular}

From top to bottom, the simulated geometry included: X-ray source, compression paddle, breast phantom, support paddle, air gap and the detector. The Source to Image Distance (SID) was $660 \mathrm{~mm}$. The detector structure, up to the amorphous selenium (aSe) surface, was included in the geometry ${ }^{12}$, the data was obtained via private communication with Hologic 
Inc. Figure 1 shows both the realistic and pencil beam geometries and Table 1 gives more details about the composition and thicknesses.

In this report, the SPSF kernels were simulated using a narrow pencil beam and a spectrum of $28 \mathrm{kVp} \mathrm{W} / \mathrm{Rh}(\mathrm{HVL}=0.538$ $\mathrm{mmAl}$ ). The photon cross-section "Electromagnetic physics option 4 package" was used both for the validation and the experiment. 20-60 runs, of $10^{9} \mathrm{X}$-ray photons each, were simulated, ensuring uncertainties lower than 5\% and calculated as suggested by Sempau et al. ${ }^{13}$.

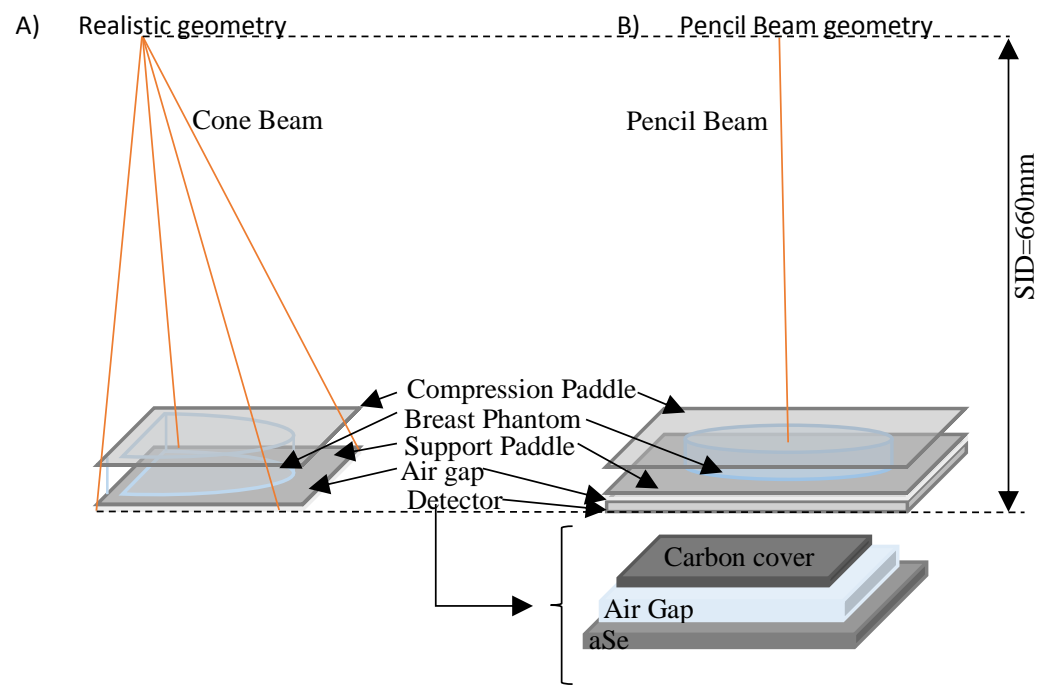

Figure 1. Diagram of a realistic geometry, used for validation purposes, (A) and of the experiment geometry (B). Figure A shows an example using a cone beam aligned with the chest wall of the breast phantom. Figure B shows the pencil beam example and a detector added after the air gap.

\subsection{Prediction of the scatter-reduced image}

To account for the difference in scattering between the breast phantom and the background areas, two different kernels were simulated, $\mathrm{K}_{\text {Phantom }}$ and $\mathrm{K}_{\text {Background }}$. For the background kernel, the pencil beam geometry was simulated with the absence of the phantom. The analysis only needed of these two kernels because of the characteristics of the chosen phantom. More kernels would need to be used if, for example, the thickness of the phantom was variable.

During the processing step, a segmentation algorithm based on thresholding was performed in order to separate both phantom and background areas, before the two convolutions were performed. The final scattering image was the sum of both convolutions, see Figure 2.

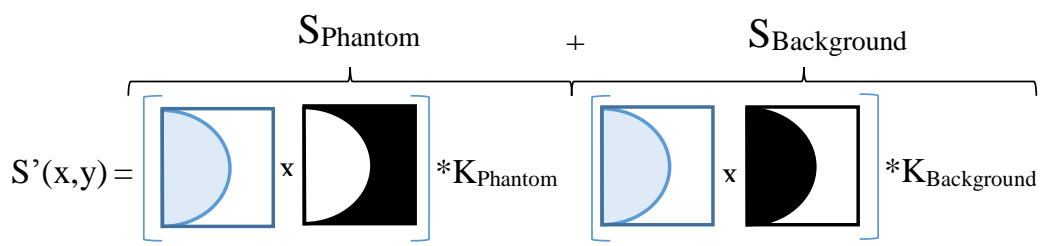

Figure 2: The schematic diagram shows how the scatter image is calculated with the pencil beam methodology. The initial (grid-less) image is multiplied by binary masks (from the segmentation algorithm) and convolved by the correspondent kernels. The final scatter image is the sum of each convolution.

The predicted primary image ( $\left.\mathrm{P}^{\prime}\right)$ was calculated by subtracting the predicted scattering image, $\mathrm{S}^{\prime}(\mathrm{x}, \mathrm{y})$, from the grid-less image. 


\section{RESULTS}

The processed grid-less image was compared to the image acquired with the use of a grid using several imaging metrics. Both images were acquired under the same acquisition conditions and dose in order to facilitate the comparison.

Before the scatter kernels were calculated, the geometry of the system and the kernel curves were validated with previous publications.

\subsection{Validation}

\section{Geometry Validation}

The values given in the report of the American Association of Physicists in Medicine (AAPM) task group 195, case 3 Mammography and breast tomosynthesis, were used as a benchmark for validating the PSF simulations ${ }^{14,15}$. The results obtained showed an average discrepancy lower than $1 \%$ when compared with the results from the AAPM report.

The geometry followed in the validation is shown in Figure 1-A. Table 1 gives details about the materials, thicknesses and positions. A combination of two X-ray beams (cone and pencil beam) and two X-ray spectra (monoenergetic and polyenergetic) were used (see Table 2 ). The spectrum values were obtained from ${ }^{14}$. For each of them, the primary radiation, and scattering radiation (single and multiple Compton and Rayleigh events) were measured in 7 different regions of interest (ROIs), as described by ${ }^{14}$. Enough particles were simulated sufficient to produce Standard Error of the Mean (SEM) values equal to or lower than $1 \%$.

Table 2. The table shows the four X-ray source/spectrum combinations used for the geometry validation of the scatter PSF code. The last column shows both the average and maximum discrepancy of the recorded events found when comparing the results with the American Association of Physicists in Medicine report - TG 195- Case 3 data ${ }^{14}$.

\begin{tabular}{|cccc|}
\hline Validation & Source type & Spectrum & $\begin{array}{c}\text { Average difference }(\%) \\
(\text { Max. })\end{array}$ \\
\hline V1 & Cone Beam & Mono energetic $(16.8 \mathrm{keV})$ & $0.82(4.0)$ \\
V2 & Cone Beam & $30 \mathrm{kVp} \mathrm{Mo} / \mathrm{Mo}(\mathrm{HVL}=0.3431 \mathrm{mmAl})$ & $0.48(3.6)$ \\
V3 & Pencil Beam & Mono energetic $(16.8 \mathrm{keV})$ & $0.37(1.6)$ \\
V4 & Pencil Beam & $30 \mathrm{kVp} \mathrm{Mo} / \mathrm{Mo}(\mathrm{HVL}=0.3431 \mathrm{mmAl})$ & $0.29(1.9)$ \\
\hline
\end{tabular}

Table 3. The table shows eight thickness and composition combinations used with a simplified geometry (phantom, air gap and ideal detector) for the SPSF validation of the scatter PSF code. The SPR values (area under the curve) for a circular area of $100 \mathrm{~mm}$ radius are compared with Diaz et $\mathrm{al}^{7}$ and Sechopoulos et al ${ }^{16}$.

\begin{tabular}{|ccccccc|}
\hline & & \multicolumn{2}{c}{ Area under the curve (SPR - 100mm radius) } & \multicolumn{2}{c|}{ Difference $(\%)$} \\
\cline { 2 - 6 } Thickness (cm) & Glandularity $(\%)$ & Sechopoulos & Diaz & Marimon & $\begin{array}{c}\text { Mar.- } \\
\text { Secho. }\end{array}$ & $\begin{array}{c}\text { Mar.- } \\
\text { Diaz }\end{array}$ \\
\hline & 0 & $5.33 \times 10^{-1}$ & $5.23 \times 10^{-1}$ & $5.36 \times 10^{-1}$ & -0.6 & 2.5 \\
5 & 50 & $5.57 \times 10^{-1}$ & $5.40 \times 10^{-1}$ & $5.54 \times 10^{-1}$ & 0.5 & 2.6 \\
& 100 & $5.92 \times 10^{-1}$ & $5.68 \times 10^{-1}$ & $5.75 \times 10^{-1}$ & 2.9 & 1.2 \\
& Water & $5.91 \times 10^{-1}$ & $5.89 \times 10^{-1}$ & $5.94 \times 10^{-1}$ & -0.5 & 0.8 \\
2 & & $2.44 \times 10^{-1}$ & $2.39 \times 10^{-1}$ & $2.43 \times 10^{-1}$ & 0.4 & 1.7 \\
4 & 50 & $4.55 \times 10^{-1}$ & $4.41 \times 10^{-1}$ & $4.55 \times 10^{-1}$ & 0.0 & 3.2 \\
8 & & $6.63 \times 10^{-1}$ & $6.40 \times 10^{-1}$ & $6.63 \times 10^{-1}$ & 0.0 & 3.6 \\
& & $8.72 \times 10^{-1}$ & $8.43 \times 10^{-1}$ & $8.72 \times 10^{-1}$ & 0.0 & 3.4 \\
\hline
\end{tabular}

\section{SPSF Validation}

The SPSFs have been validated against the data published by Diaz et al. ${ }^{7}$ and Sechopoulos et al ${ }^{16}$. 
Table 3 shows the comparison with the previous published data, the results show excellent agreement with both Diaz and Sechopoulos. In this geometry the compression and support paddles were removed, leaving only a cylindrical phantom and an air gap between the phantom and an ideal detector. For all the cases, an energy spectrum of $26 \mathrm{keV} \mathrm{Mo} / \mathrm{Mo}$ and an air gap of $10 \mathrm{~mm}$ was used.

\section{CIRS 010A Phantom: Grid vs. post processed grid-less images}

The post-processed grid-less image, $\mathrm{P}$ ' equation (3), and the grid image were compared in terms of uniformity and Contrast to Noise Ratio (CNR).

\section{Uniformity}

A comparison of the unprocessed grid-less image (I), the processed grid-less image (P') and the grid image is shown in Figure 3 .
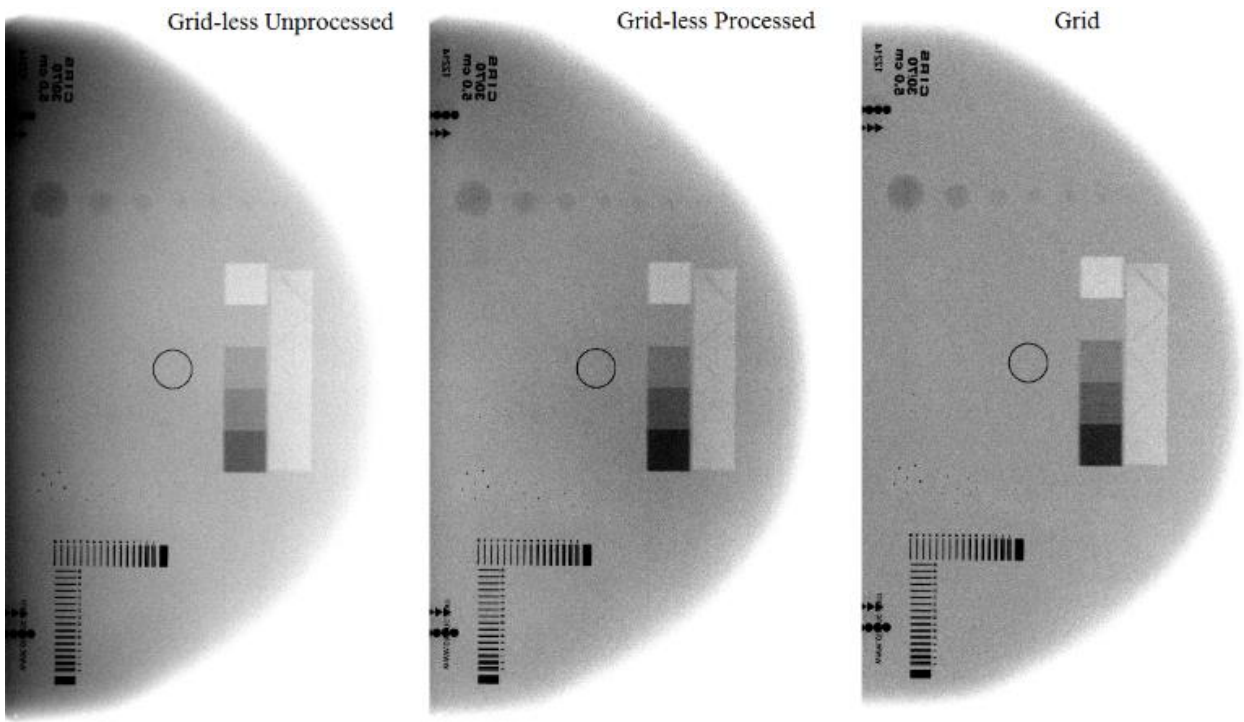

Figure 3. This figure shows the phantom images obtained without a grid (left), the processed grid-less image (centre) and the image captured with an anti-scatter grid (right).

A horizontal profile, as highlighted in Figure 4, has been plotted for the three images.
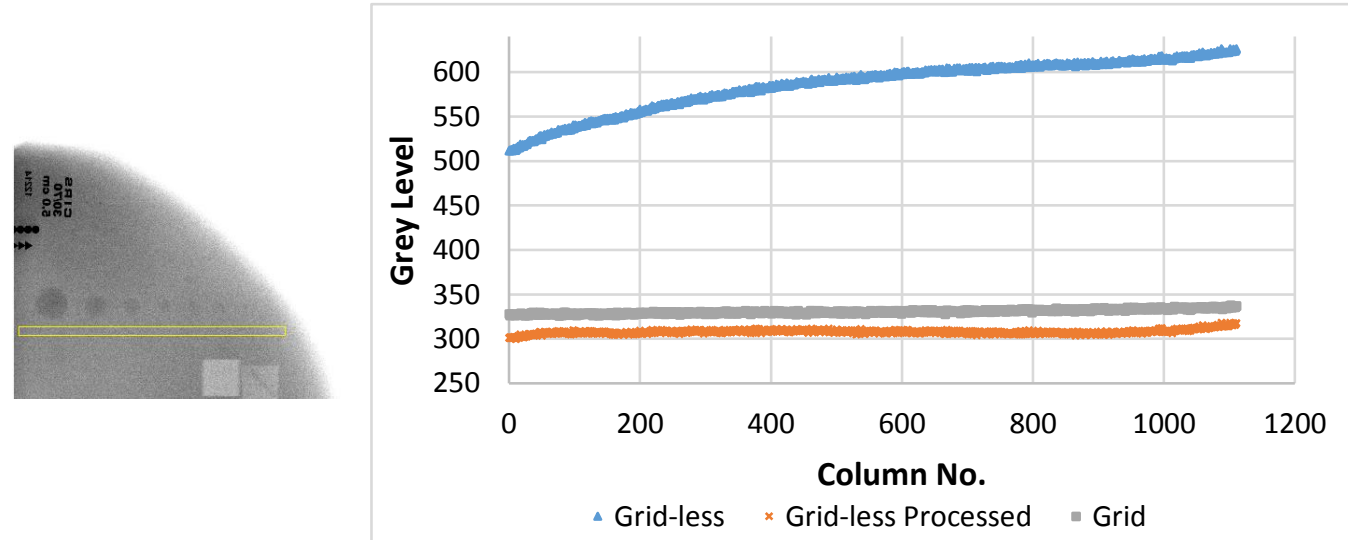

Figure 4. The figure shows the profile plots of the grid-less, processed grid-less and grid images for the area highlighted in the yellow rectangle (left). 


\section{Contrast to noise ratio}

The CNR was calculated following equation (5). The area analysed corresponds to the area surrounding the step phantom, the equation was applied to the 5 different regions of interest (ROIs), see Figure 5-left.

$$
C N R=\frac{\bar{x}_{R O I}-\bar{x}_{\text {background }}}{\sigma_{\text {background }}},
$$

where,

$\bar{x}_{R O I}$ and $\bar{x}_{\text {background }}$ are the mean pixel value of the object and background ROI, respectively.

$\bar{\sigma}_{\text {background }}$ is the standard deviation of the background ROI.
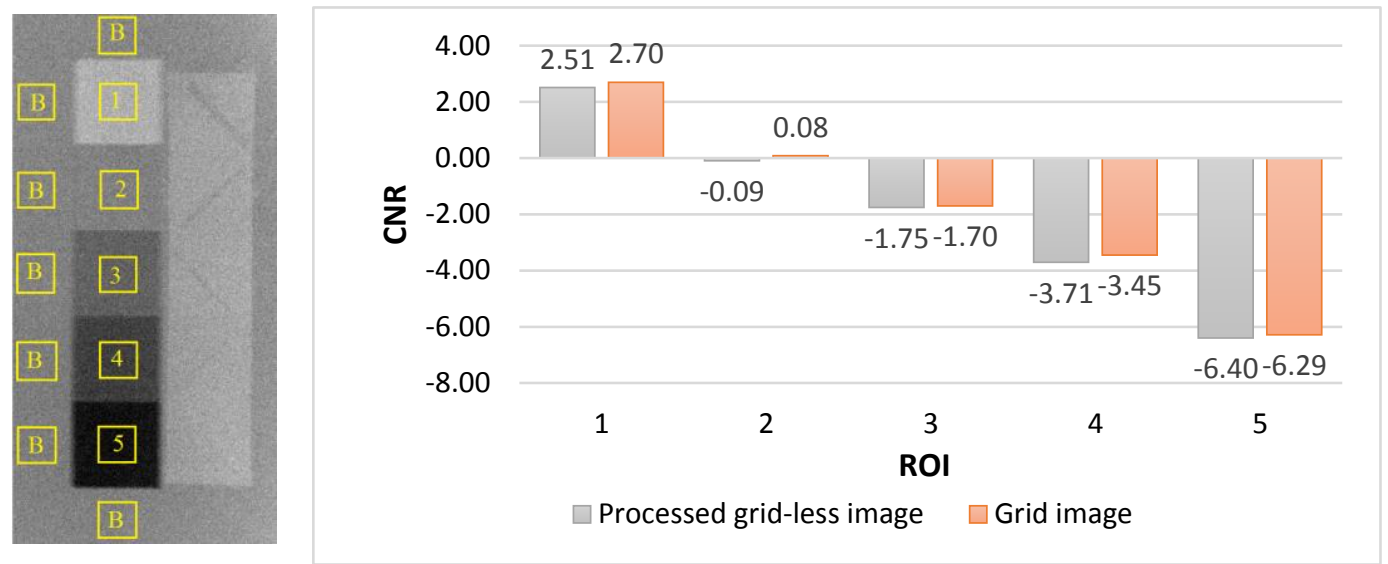

Figure 5. The right figure show a plot comparing the CNR of the processed grid-less image and the image acquired with an anti-scatter grid. The CNR was calculated using the step phantom area of the images. The figure on the left shows the ROI areas used in the calculation (numbered 1 to 5) and the background regions (B). For the background, the values of the seven boxes were averaged.

\section{DISCUSSION}

The analysis with this convolution-based technique shows an overall improvement of the processed grid-less image over the unprocessed grid-less image, and comparable results when compared with the grid image.

In terms of uniformity, the unprocessed grid-less image presents a non-uniformity higher than 100 grey values. This nonuniformity is reduced by more than $85 \%$ in the processed grid-less image. When compared to the grid image, it is possible to see that the image is flatter in the central region (5 vs. 10 grey values).

Towards the edges of the image, the non-uniformity of the processed image increases. In future measurements, further corrections should be applied around these areas, as suggested by Diaz et al. ${ }^{7}$. Towards the chest-wall area, the discrepancy is probably introduced by the effect that the plastic holder of the phantom (outside the collimated area) introduces in the scatter.

The CNR measurements give comparable results to those obtained with the grid image. For the first ROI of the step phantom the CNR is slightly lower, while ROIs 2-5 show an improvement.

An improvement in the overall result is expected for real mammographic images. The objects that can be found inside the phantom present an extreme case, covering different ranges of materials and introducing differences in the scattering. Taking the step phantom area, for example, the steps go from 0 to $100 \%$ glandular tissue. These differences are not taken into account in the convolution, as only one kernel that matches the phantom general material is used. 


\section{CONCLUSIONS}

Measurements made with a commercial planar mammography system were used with convolution-based image scatter reduction techniques in an attempt to generate images with high contrast to noise ratio, without the use of an anti-scatter grid.

Analysis of the images showed that the quality of the predicted primary image is comparable to the grid image in terms of uniformity and presents general improvements in the contrast to noise ratio. The results are expected to show further improvement when using more clinically-representative phantoms.

Further studies will include more realistic phantoms and a more complex analysis of the breast edge areas to gauge the level of complexity required to build the scatter point spread functions in a real system. The results obtained so far indicate that future grid-less mammography systems will be able to reduce the dose delivered to the patient without a compromise in the image quality.

\section{ACKNOWLEDGEMENTS}

This work has been done in collaboration with PerkinElmer. The authors would like to thank FilmLight Ltd for their donation of computer resources, the medical physics department of the Barts Health NHS Trust for the access to their mammography system, where the images under study where acquired, and Dr. Ioannis Sechopoulos, for a helpful discussion about this work.

Oliver Díaz is supported by the European Union within the Marie Sklodowska-Curie Innovative Training Networks (H2020-MSCA-IF-2014 SCARtool project, reference 657875) and the Ministry of Economy and Competitiveness of Spain, under project reference DPI2015-68442-R.

\section{REFERENCES}

[1] Wang, A. et al., "Asymmetric scatter kernels for software-based scatter correction of gridless mammography," SPIE 9412, 1I-1-7 (2007).

[2] Boone, J. M. and Cooper, V. N., "Scatter/primary in mammography: Monte Carlo validation,” Medical Physics 27(8), 1818-1831 (2000).

[3] Ducote, J. L. and Molloi, S., "Scatter correction in digital mammography based on image deconvolution," IOPPhysics in Medicine and Biology 55, 1295-1309 (2010).

[4] Binst, J. et al., "Evaluation of automated CDMAM readings for non-standard CDMAM imaging conditions: Gridless acquisitions and scatter correction," Oxford University Press - Radiation Protection Dosimetry, 1-4 (2015).

[5] Krol, A. et al., "Scatter reduction in mammography with air gap," Medical Physics 23(7), 1263-1270 (1996).

[6] Diaz, O. et al., "A fast scatter field estimator for digital breast tomosynthesis," Proc. SPIE 8313, Medical Imaging (2012).

[7] Diaz, O. et al., "Estimation of scattered radiation in digital breast tomosynthesis," IOP Physics in Medicine and Biology 59, 4375-4390 (2014).

[8] Love, L. A. and Kruger, R. A., "Scatter estimation for a digital radiographic system using convolution filtering," Medical Physics 14(2), 178-185 (1987).

[9] Allison, J. et al., "GEANT4 developments and applications,” IEEE Transactions on nuclear science 33(1), 270278 (2006).

[10] Feijó, P. V. and Hogg, G., "Geant4 validation on mammography applications," Proc. IEEE Nuclear Science symposium and Medical Imaging (2008).

[11] Diaz, O., [Scattered radiation in projection X-ray mammography and digital breast tomosynthesis], PhD Thesis University of Surrey (2013).

[12] Marimon, E., Diaz, O. et al., "Detailed analysis of scatter contribution from different simulated geometries of X-ray detectors," IWDM Proceedings, 203-210 (2016).

[13] Sempau, J., Sánchez-Reyes, A. and Salvat, F., "Monte Carlo simulation of electron beams from an accelerator head using PENELOPE," Physics in medicine and biology 46(4), 1163-1186 (2001). 
[14] Sechopoulos, I. et al., "The report of AAPM task group 195: Monte Carlo reference data sets for imaging research," AAPM (2015).

[15] Sechopoulos, I. et al., "Monte Carlo reference data sets for imaging research: Executive summary of the report of AAPM research committee task group 195," Medical Physics 42, 5679 (2015).

[16] Sechopoulos, I. et al., "Scatter radiation in digital tomosynthesis of the breast," Medical Physics 34(2), 564-576 (2007). 